There was no history of a foreign body in the nose at any time before.

Anterior rhinoscopy showed a rough dark stony mass covered with greenish discharge. Probing gave a metallic sound. Postnasal examination showed a greenish discharge. A clinical diagnosis of a rhinolith was made. Radiology showed the rhinolith's position (Fig. 1) in the floor of the right nostril with mucosal thickening of the right maxillary antrum. The nasal swab showed a growth of $B$. proteus, sensitive

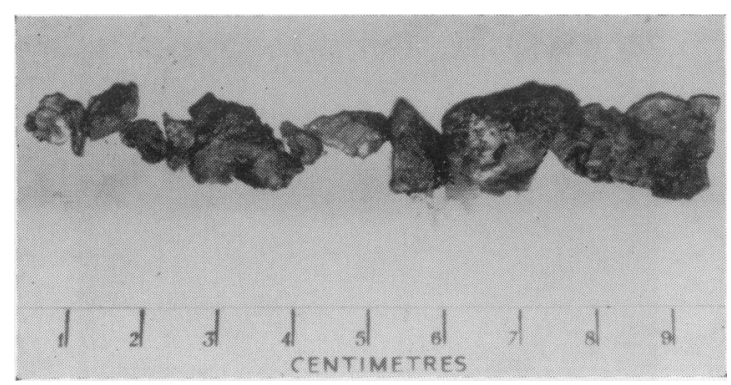

FIG. 2. Photograph of the pieces after removal. to streptomycin, chloramphenicol and ampicillin.

This stone was removed in pieces under general anaesthesia (Fig. 2). Small pieces were fished out from the postnasal space. The nasal cavity was washed out with normal saline.

The patient was given antibiotics for 7 days. She had an uneventful recovery after the operation.

The stone weighed $4.4 \mathrm{~g}$; chemical analysis showed that it consisted of calcium and magnesium, oxalates and urates.

\section{Acknowledgments}

I acknowledge with pleasure and gratitude the help given by Mr H. Wickham, Mr J. A. Kersley and Mr A. Robinson.

\section{References}

AbU-Jaudeh, C.N. (1951) A giant rhinolith. Laryngoscope, 61, 27.

HuNT, W.L., CotTon, B.P. \& JoSEPH, D.J. (1966) Rhinoliths. Arch. Otolaryng. 83, 256.

MYERSON, M.C. (1928) Rhinolith requiring external nasal operation for its removal. Laryngoscope, 38, 393.

PoLsON, C.J. (1943) On rhinoliths. J. Laryng. 58, 79.

VAN AlYeA, O.E. (1952) Rhinolith: a report of two cases. Ann. Oto-laryng. (Paris), 61, 490.

\title{
Unusual presentation of intralobar pulmonary sequestration
}

\author{
Brig. H. B. LAL \\ F.R.C.P.(E), D.C.H. \\ Consultant in Medicine
P. N. Dhawad
M.B.B.S.
Registrar

\author{
A. L. BAHL \\ M.D. \\ Assistant Surgeon
}
A. K. Sharma
M.S.
Registrar

\author{
S. MUKHERJEE \\ F.R.C.S.(Eng.), F.R.C.S.(Edin.), \\ F.A.C.S. \\ Associate Professor of Surgery \\ G. S. SARIN
R.C.P.(Canada)
Senior Radiologist
}

Departments of Medicine, Surgery and Radiology, Willingdon Hospital, New Delhi, India

BRONCHOPULMONARY sequestration was first described adequately by Pryce (1946), only three such case reports having been published till then. A total of about 150 surgically treated cases had been reported up till 1966 (Rubin et al., 1966). The intralobar variety consists of a bronchopulmonary mass or cyst enveloped in the same pleura as the normal lung and has an abnormal blood supply by an aberrant vessel originating from the aorta in the vicinity of the diaphragm. This ectopic pulmonary mass does not communicate with the normal bronchial tree. In the extralobar type, the lung mass is completely separate from the lung and has no connection with the arteries and veins of the pulmonary circulation (James \& Cuthbert, 1967)

Pulmonary sequestration is often detected on operation or autopsy although pre-operative diagnosis has been made by aortography (Turk \& Lindskog, 1961 ; Sutton \& Samuel, 1963 ; Ranniger \& Valvassori, 1964 ; Rubin et al., 1966). The clinical diagnosis in most of these patients is empyema, lung abscess, bronchiectasis, or recurrent pneumonia (Borrie, Lichter \& Rodda, 1963). The case described below is of interest because of its unusual mode of presentation.

\section{Case report}

V.K., a 16-year-old male, was admitted on 7 December 1966 with a history of fever of 
8 days' duration. The patient never had cough, expectoration, haemoptysis or chest pain.

Examination revealed an ill patient with a pulse rate of $140 / \mathrm{min}$, temperature $104^{\circ} \mathrm{F}$, and dyspnoea. Clubbing, cyanosis and lymphadenopathy were absent. The trachea and cardiac apex were deviated to the right, with dullness in left infrascapular area and diminished breath sounds, vocal fremitus and resonance. There was no other abnormality.

Investigations: A marked polymorphonuclear leucocytosis, moderate anaemia and elevated erythrocyte sedimentation rate were present. Urine and blood cultures were sterile. Chest X-ray confirmed the tracheal deviation and showed two rounded opacities in the left lung, the upper one with an air-fluid level (Fig. 1). These opacities appeared to be in the line of the inter-lobar fissure.

Progress: An interlobar hydropneumothorax was suspected and a thoracic surgeon consulted. A needle was inserted in the line of the interlobar fissure in the mid-axillary line and $500 \mathrm{ml}$ of purulent fluid aspirated which was sterile on culture. A repeat chest roentgenogram revealed that the upper opacity had been completely evacuated, while the lower one had an airfluid level (Fig. 2). The patient was treated with heavy doses of crystalline penicillin $(4 \mathrm{mega}$ units/day). A dramatic improvement in his condition was observed within 2 days with the dis-

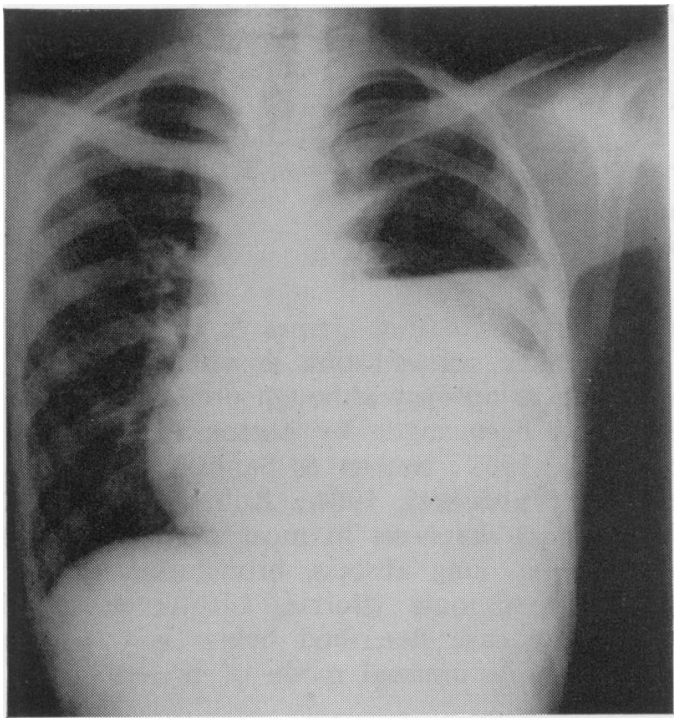

FIG. 1. P.A. view of chest X-ray on admission showing tracheal deviation and two rounded opacities, the upper one with an air-fluid level.

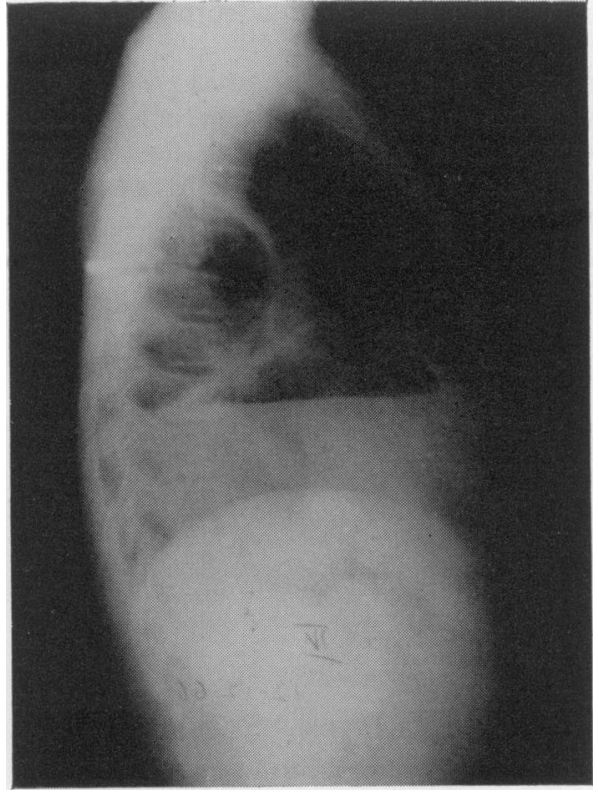

FIG. 2. Lateral view of the chest after aspiration.

appearance of fever and toxaemia. Another aspiration was attempted after 4 days, which yielded $250 \mathrm{ml}$ of pus. No change in the roentgenogram was, however, observed. Another X-ray of chest repeated on 2 February 1967 made evident the underlying parenchymal disease, viz. cystic disease of the lung. A bronchogram performed the next day showed cystic areas in the lower lobe of the left lung, which remained unfilled with the dye and displaced the normal bronchial tree.

A left lower-lobe lobectomy was performed by S.M. A large aberrant vessel arising from the aorta was seen and ligated. This supplied the sequestrated bronchopulmonary segment. The patient made an uneventful recovery and was well on 30 June 1967. Injection of the contrast medium under pressure into the bronchus of the resected lung failed to outline the bronchi. The pathological examination revealed a mass occupying most of the lower lobe. On section of the specimen, a large cyst containing inspissated pus was seen with mutiple small cysts in the surrounding areas.

\section{Discussion}

Radiologically, the sequestrated segment appears as a dense, sharply demarcated mass which is liable to be mistaken for suppurative pneumonia or a tumour (Foster-Carter, 1963). This case presented as an interlobar pyopneumo- 
thorax possibly because of two large cysts getting infected. The anomaly is often present in men on the left side (Claman \& Ehrenheft, 1960 ; Rubin et al., 1966) as in this patient. The aberrant vessel supplied only the sequestrated mass. Pryce (1946) reported that this vessel may supply only the sequestrated segment, the normally connected lung or this segment along with adjoining area of normal lung. The possible existence of an anomalous vessel must be kept in mind while operating on cystic lung, otherwise the division of a supposed stout adhesion may cause fatal haemorrhage (Douglas, 1948). The aetiology of this condition is one upon which few investigators agree. The various theories of origin are summarized by Boyd (1953) and Boyden (1958).

\section{References}

Borrie, J., Lichter, I. \& RodDA, R. (1963) Intralobar pulmonary sequestration. Brit. J. Surg. 50, 623.

BoYD, G. (1953) Intralobar pulmonary sequestration. Dis. Chest, 24, 162.
BOyden, E.A. (1958) Bronchogenic cysts and the theory of intralobar sequestration. New embryologic data. J. thorac. Surg. 35, 604.

Claman, M.A. \& Ehrenheft, J.L. (1960) Bronchopulmonary sequestration. J. thorac. cardiovasc. Surg. 39, 531.

Douglas, R. (1948) Anomalous pulmonary vessels. J. thorac. Surg. 17, 712.

Foster-CARTER, A.F. (1963). In: Chest Diseases (Ed. by K. M. A. Perry and T. M. Sellors), Vol. I. Butterworths, London.

JAmes, W.B. \& Cuthbert, R.J. (1967) Intralobar pulmonary sequestration. Postgrad. med. J. 43, 359.

PryCe, D.M. (1946) Lower accessory pulmonary artery with intralobar sequestration of lung: A report of seven cases. J. Path. Bact. 58, 457.

RanNiger, K. \& Valvassori, G.E. (1964) Angiographic diagnosis of intralobar pulmonary sequestration. Amer. $J$. Roentgenol. 92, 540.

Rubin, E.H., Rubin, M., Attai, L. \& Heimann, W.G. (1966) Intralobar pulmonary sequestration: Aortographic demonstration. Dis. Chest, 50, 561.

Sutton, D. \& SAmuel R.H. (1963) Thoracic aortography in intralobar sequestration. Clin. Radiol. 14, 317.

TURK, L.N. \& Lindskog, G.E. (1961) The importance of angiographic diagnosis in intralobar pulmonary sequestration. J. thorac cardiovasc. Surg. 41, 299.

\section{Unusual method of diagnosis in a case of benign duodenocolic fistula}

\author{
J. H. P. Cuddigan \\ B.A., M.B., B.Ch. \\ Medical Registrar
}

\author{
M. A. EDgar \\ M.A., M.B., B.Ch. \\ Senior Surgical House Officer
}

St Peter's Hospital, Chertsey, Surrey

FIRST described by Sanderson in 1863, benign duodenocolic fistula is relatively rare, and only thirty-one cases have been described subsequently.* The condition is usually diagnosed from barium studies of the duodenum or, more commonly, the colon. This paper describes a case in which the diagnosis was made as a result of attempted jejunal biopsy, having been missed by conventional radiographic methods. The literature has been reviewed and the possible causes of the disease are discussed.

\section{Case report}

A 65-year-old woman had attended her own doctor with a 6-week history of diarrhoea, occasional vomiting and weight loss. The symptoms

\footnotetext{
*Seven cases of duodenocolic fistula complicating gallbladder disease have been described but excluded from the present review as they were really cholecysto-duodenocolic fistula.
}

had originally been thought due to acute gastroenteritis and she had been treated at home with antibiotics. However, the symptoms recurred and persisted for 2 months and she was admitted for investigation.

She had suffered occasional epigastric discomfort for some years, relieved by antacids. For 3 years she had been treated for hypertension with reserpine and Ismelin-Navidrex $K$. She had had no pain during her present illness and described her motions as loose, but not pale, and free from blood or mucus.

On examination, she was not clinically dehydrated but appeared mildly anaemic. Recent weight loss was apparent ; the abdomen appeared slightly distended and bore a mid-line hysterectomy scar. Further physical examination was normal.

Investigations: $\mathrm{Hb} 76 \%(11 \cdot 1 \mathrm{~g} / 100 \mathrm{ml})$; normal white blood cells and platelets ; MCHC 33\% ; ESR $15 \mathrm{~mm} / \mathrm{hr}$, serum iron $50 \mu \mathrm{g} / 100 \mathrm{ml}$; 